\title{
Microwave rotation-tunneling spectroscopy of the water-methanol dimer: Direct structural proof for the strongest bound conformation
}

\author{
Paul A. Stockman a) and Geoffrey A. Blake \\ Division of Geological and Planetary Sciences, California Institute of Technology, Mail Stop 150-21, \\ Pasadena, California 91125 \\ Frank J. Lovas and Richard D. Suenram \\ National Institute of Standards and Technology, Optical Technology Division, Mail Stop Physics B208, \\ Gaithersburg, Maryland 20899
}

(Received 24 March 1997; accepted 9 June 1997)

\begin{abstract}
Rotation-tunneling $a$-type spectra of $\mathrm{CH}_{3} \mathrm{OH} \cdots \mathrm{H}_{2} \mathrm{O}$ and $\mathrm{CH}_{3} \mathrm{OD} \cdots \mathrm{D}_{2} \mathrm{O}$ were recorded between 18 and $60 \mathrm{GHz}$ using direct absorption microwave spectroscopy, and for $\mathrm{CH}_{3} \mathrm{OH} \cdots \mathrm{H}_{2} \mathrm{O}$, ${ }^{13} \mathrm{CH}_{3} \mathrm{OH} \cdots \mathrm{H}_{2} \mathrm{O}, \mathrm{CH}_{3} \mathrm{OH} \cdots \mathrm{DOH}, \mathrm{CD}_{3} \mathrm{OH} \cdots \mathrm{H}_{2} \mathrm{O}$, and $\mathrm{CH}_{3} \mathrm{OD} \cdots \mathrm{D}_{2} \mathrm{O}$ between 7 and $24 \mathrm{GHz}$ using a Fourier-transform microwave spectrometer. Because $\mathrm{CH}_{3} \mathrm{OH}$ and $\mathrm{H}_{2} \mathrm{O}$ are capable of both accepting and donating hydrogen bonds, there exists some question as to which donor-acceptor pairing of the molecules is the lowest energy form. This question is further emphasized by the ambiguity and variety present in previous experimental and computational results. Transitions arising from the methyl torsional $A$ state were assigned in each of the studied isotopomers, and for the $A$ and $E$ states in $\mathrm{CH}_{3} \mathrm{OH} \cdots \mathrm{H}_{2} \mathrm{O}$. While the measured components of the dipole moment for the parent $\left(\mathrm{H},{ }^{12} \mathrm{C},{ }^{16} \mathrm{O}\right)$ isotopomer- $\mu_{a}=7.956 \pm 0.03 \times 10^{-30} \mathrm{C} \mathrm{m} \quad(2.385 \pm 0.008 \mathrm{D}), \quad \mu_{b}=3.636$ $\pm 0.02 \times 10^{-30} \mathrm{C} \mathrm{m}(1.090 \pm 0.006 \mathrm{D}), \mu_{c}=0.43 \pm 0.47 \times 10^{-30} \mathrm{C} \mathrm{m}(0.13 \pm 0.14 \mathrm{D})$, where the errors correspond to $1 \sigma$ uncertainties - are consistent with either conformation, the fit of the structure to the rotational constants demonstrates unambiguously that the lower-energy conformation formed in supersonically cooled molecular beams corresponds to a water-donor, methanol-acceptor complex. The results and implications for future work are also discussed in terms of the permutation-inversion theory presented by Hougen and Ohashi [J. Mol. Spectros. 159, 363 (1993)]. @ 1997 American Institute of Physics. [S0021-9606(97)02134-X]
\end{abstract}

\section{INTRODUCTION}

Due to the amphoteric nature of hydroxyl groups with respect to hydrogen bonding, methanol and water can act as both hydrogen bond donors and acceptors. When they are clustered together in the form of a gas-phase heterodimer, then there can exist two forms of the dimer which are structurally, and presumably energetically, distinct: a methanoldonor, water-acceptor form; and a water-donor, methanolacceptor one. Determining which of the two forms has a greater binding energy has been the subject of several computational and experimental studies. However, no consensus is available from the calculations, which fall evenly on both sides of the question, and the results from the experiments are indirect and sometimes complicated by measurements taken in the condensed phase. We offer here direct structural proof for the lowest-energy form of the water-methanol dimer obtained from microwave rotation-tunneling spectroscopy of jet-cooled water-methanol dimers.

Methanol and water are two of the most common solvents in chemistry, and understanding their intermolecular hydrogen bonding potentials is necessary in order to correctly model important bulk solvent-solute dynamics. When mixed together, the water-methanol solvent system is completely miscible in all proportions and is widely used as the supporting liquid phase in liquid chromotography and other

a)Present address: The BOC Group, Murray Hill, NJ 07974. solvent-based separation techniques. The water-methanol dimer is the latest, and one of the most chemically complex, in a series of water-containing, hydrogen-bonded dimers studied in our laboratories by microwave and far-infrared spectroscopy, for the purpose of fitting the observed spectra to intermolecular potential energy surfaces (IPSs). ${ }^{1-11}$ The complex formed between methanol and water is particularly interesting because both hydrophobic and hydrophilic forces contribute strongly to the IPS of this relatively small dimer, creating a high degree of anisotropy. In addition, the watermethanol dimer will provide a test of the adaptability of the IPSs of simpler dimers to larger, multi-functional interactions.

Mixed solvent systems are inherently more difficult to model than single component ones, and increase the types and complexity of possible solvent-solute and solventsolvent interactions. This is especially easy to envision for the water-methanol system, where $\mathrm{CH}_{3} \mathrm{OH}$ has the propensity to accept two hydrogen bonds but donate only one. Moreover, even though the water-methanol system is completely miscible in all proportions, the solution resulting from the mixing of the two liquids is not necessarily homogeneous. Using statistical mechanics methods, Matsumoto and co-workers simulated the bulk structure of different mixtures of methanol and water. ${ }^{12}$ Not surprisingly, the hydrophobic methyl groups caused the methanol to prefer (near-) surface sites, and the interactions were strong enough to cre- 
ate a net alignment of methanol molecules in these enriched surface regions. The effect is particularly dramatic for solutions with low molar volumes of methanol which are shown to have most of the methanol pooling in such near-surface volumes. This picture is reinforced by kinetic studies which show that the free energy of mixing of methanol and water is driven by excess entropy, not excess enthalpy. ${ }^{13}$

By far the most studied polyatomic...polyatomic hydrogen-bonded complex, both computationally and experimentally, is the water-water dimer. ${ }^{14,4,3,15-20}$ The watermethanol dimer provides a heteromolecular analog to this well-studied system, not only to the IPS being developed for this complex but also to the permutation-inversion and vibration-rotation-tunneling formalisms created especially for this dimer. ${ }^{21,1,22}$ Of course, methanol is chemically much different from water, but the dynamics of the unsubstituted position, whether it turns out to be donor or acceptor, should be largely conserved if the present IPS and formalism for the water-water dimer are correct and adaptable to other hydroxyl-hydroxyl interactions. The water-methanol complex also provides the opportunity to synthesize an IPS from two other previously determined IPSs without the perturbing effects of three-body forces encountered in previous attempts.

For example, Elrod et al. have studied the $\mathrm{Ar}_{2} \cdots \mathrm{DCl}$ complex to determine how well two-body IPSs sum to give the full three-body IPS. ${ }^{23}$ They have found that three-body forces contribute a very significant $8 \%$ to the overall energetics of the complex. This complication should be avoidable for synthesizing the IPS of water-methanol, a two-body interaction, from the water-water and methane-water potentials. Studies of $\left(\mathrm{H}_{2} \mathrm{O}\right)_{2}$ and $\left(\mathrm{CH}_{4} \cdots \mathrm{H}_{2} \mathrm{O}\right)$, investigated experimentally by Dore et al. ${ }^{24}$ provide the basis for such a comparison during the ongoing research on each of these complexes. While three-body and larger-body contributions to IPSs are definitely the rule and not the exception in the study of solution dynamics, the approach outlined above for the water-methanol dimer has relevance to the modeling of large biomolecular interactions, such as protein folding. Here, the complexity of the system derives not from a myriad of small solvent interactions, but rather the forces between polyfunctional sidegroups along one backbone give the protein a high degree of order. Some protein folding approaches already use a matrix of dimeric, albeit crude, IPSs to form intramolecular potentials. The water-methanol dimer will be a good test of this method and will also provide refined potentials for its application.

Our initial assumption in considering the two possible hydrogen-bonded forms of the water-methanol dimer is that the hydrogen bond in both forms will be geometrically similar to that of the water dimer (see Fig. 1) given by Odutola and Dyke. ${ }^{14}$ We will use the abbreviations MW and WM to refer to a specific donor-acceptor conformation and the generic notation water-methanol dimer when the structure is not specified. MW is formed from the water dimer structure by replacing the nonbonded water donor hydrogen with a methyl group; WM is formed by substituting methyl for one of the water dimer acceptor hydrogens. These are shown in

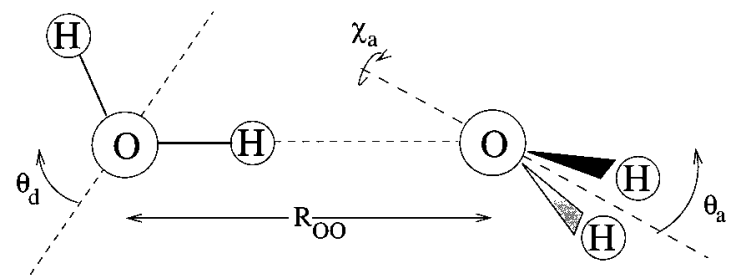

FIG. 1. Odutola and Dyke water dimer structure I: $R_{\mathrm{OO}}=2.9768 \AA$, $\theta_{d}=-53.0^{\circ}, \theta_{a}=59.7^{\circ}$, and $\chi_{a}=0.3^{\circ}$.

Figs. 2(a) and 2(b), respectively. In this framework, the MW structure has a $C_{s}$ plane of symmetry, while the WM structure has no point group symmetry elements other than the identity operation.

Although a priori the structure for the water-methanol complex is not known, the MW and WM structures do have a number of commonalities that allow some prediction of the major features of the microwave spectra. First of all, the complex is asymmetric, meaning that the asymmetry-split $K_{a}$ degeneracies will be resolvable. Also, the complex should have significant dipole moments along its $a$ and $b$ axes. A WM structure will have a small $c$ component as well; the $c$ component for MW should be zero by symmetry. Along with the fact that the structural $A$ rotational constant is between 20 and $35 \mathrm{GHz}$, both $a$-type and $b$-type transitions should be observable in the present microwave experiments. We can also anticipate complicated internal rotor effects from the methyl top, in which the angular momentum of the top couples with the overall rotation of the complex.

Exchange of identical nuclei by rotation and quantum mechanical tunneling leads to splitting of rovibrational energy levels, and the effects are often detectable with the high
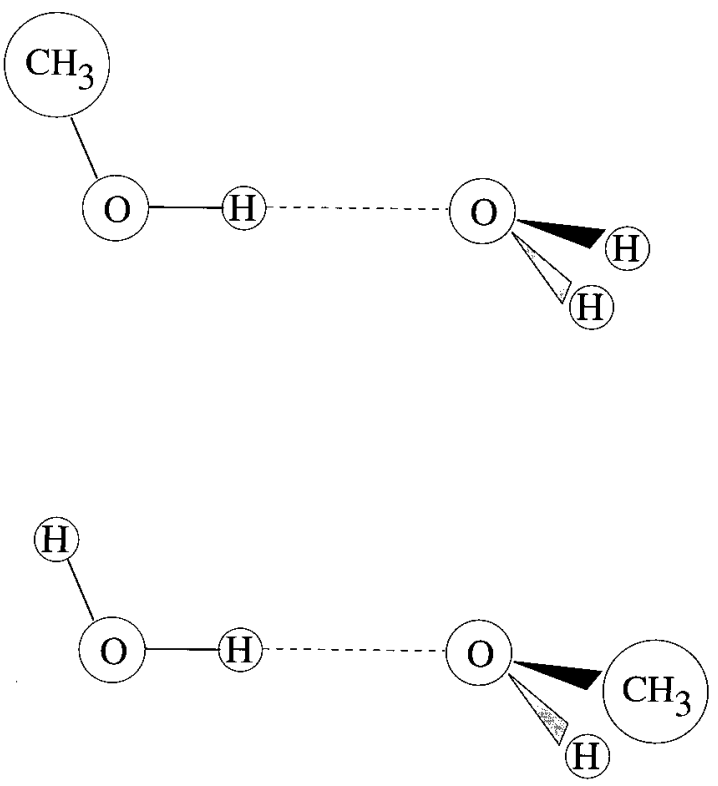

FIG. 2. Structural frameworks for (a) MW and (b) WM based on the water dimer structure. 
resolution methods employed here. By counting the number of nonsuperimposible frameworks and the feasible exchange pathways between them, one can estimate the number and magnitude of splittings to each rovibrational transition.

The two different conformations for the water-methanol dimer each generate a set of unique tunneling frameworks, and we need to consider sets of equivalent nuclei and their exchange pathways for both cases. In each structure, the methyl group has three equivalent hydrogens which can interchange about its $C_{3}$ axis. Each $\mathrm{H}_{2} \mathrm{O}$ subunit has two hydrogens which can exchange about the water $C_{2}$ axis and, in the case of WM, about the water $c$ axis. Also exchangeable are the two oxygen lone pair binding sites on each acceptor oxygen atom. Noteworthy here is that the entity which is exchangeable is the binding site, not the electrons which occupy the lone pair orbitals. The acceptor oxygen atom can be thought of as a potentially chiral center: in the case of MW, the oxygen is not chiral because the two hydrogen-bonding sites are equivalent; in WM, however, the oxygen is chiral with four different substituents- $-\mathrm{H}, \mathrm{CH}_{3}, \cdots \mathrm{H}_{2} \mathrm{O}$, and an unbound lone pair-arrayed in a near tetrahedral geometry. Hence, the chirality of the hydrogen-bonded oxygen doubles the number of nonsuperimposable frameworks for WM. The total number of such frameworks, then, is 6 for MW and 12 for WM.

Hougen and Ohashi have made a detailed permutationinversion theory investigation of the water-methanol dimer in the $K_{a}=0$ rotational manifold, and we refer to their numbering of the frameworks. ${ }^{25}$ They begin by evaluating the feasibility for each of the tunneling paths $(1 \rightarrow n)$ in the two different conformers, based on previous results for other van der Waals complexes. For MW, they estimate that the water $C_{3}$ tunneling $(1 \rightarrow 4)$ has a splitting of $\sim 3 \mathrm{~cm}^{-1}$, and that the methyl torsion $(1 \rightarrow 2,3)$ is $\sim 0.06 \mathrm{~cm}^{-1}$; the combination modes are deemed unfeasible. The considerations for WM, with twice as many possible tunneling pathways, are substantially more complex, with all feasible tunneling splittings having similar estimated values of $0.04-0.09 \mathrm{~cm}^{-1}$. Those deemed feasible are the methyl torsion $(1 \rightarrow 2,3)$, exchange of lone pairs on the acceptor $(1 \rightarrow 4)$, exchange of donor protons $(1 \rightarrow 7)$, and simultaneous exchange of both acceptor lone pairs and donor protons $(1 \rightarrow 10)$. Hougen and Ohashi also develop a flexible coordinate system in which all of these motions can be parameterized to derive the matrix elements for tunneling splittings and the selection rules for $a$-type spectra. These rules predict "top-to-top, bottom-tobottom" for the $a$-type spectra, which means that the tunneling splittings will not be easily measurable using the lowestfrequency $a$-type MW data. They also predict a total of four rotation-tunneling components for MW, and eight for WM.

Five computational studies of the water-methanol complex have been performed, and the results for the binding energy of both MW and WM minima are summarized in Table I. ${ }^{26-30}$ Taken as whole, they fall 3:2 in favor of the MW global minimum, and there are results which find the minima separated by $\sim 1 \mathrm{kcal} / \mathrm{mol}$ for both conclusions. In all fairness, however, these calculations were performed over the span of two decades, with increasingly sophisticated
TABLE I. Summary of $a b$ initio results for methanol-water dimer. Binding energies are $D_{e}$ in $\mathrm{kcal} / \mathrm{mol}$.

\begin{tabular}{llll}
\hline \hline \multicolumn{1}{c}{ Authors } & MW & WM & Ref. \\
\hline Del Bene & 6.26 & 5.22 & 26 \\
Bolis et al. & 7.5 & 7.0 & 28 \\
Tse, Newton, and Allen & 5.55 & 5.73 & 27 \\
Bakkas et al. & 5.517 & 5.433 & 31 \\
Kim, Jhon, and Scheraga & 6.74 & 7.78 & 29 \\
\hline \hline
\end{tabular}

methods and computational resources, and the findings of the studies are correlated with basis set size, with the more flexible wave functions determining that the WM structure is more stable. At the highest level of theory performed to date, the work of Bakkas et al. ${ }^{30}$ places the minima within only $0.1 \mathrm{kcal} / \mathrm{mol}$ of each other. Notwithstanding, it is clear that experiments are needed to determine the most stable conformation of the water-methanol dimer.

Several researchers have presented reasonable interpretations of vibrational spectra for isolated water-methanol complexes. Bakkas and co-workers first examined the complex in a nitrogen matrix and observed the spectral changes for all 3 intramolecular water modes and 10 out of 12 modes for methanol. ${ }^{30}$ By measuring a distinctive red shift for the methanol $\mathrm{O}-\mathrm{H}$ stretch and a corresponding blue shift of the $\mathrm{C}-\mathrm{O}$ stretch, they concluded that the complex was MW. $A b$ initio calculations performed by the authors at the MP2 $6-31 G^{* *}$ level with harmonic frequency approximations confirmed the basic spectral shifting pattern from the experiments. When the authors followed this work with a similar matrix isolation experiment in argon, the results were completely different, with spectral shifts in argon supporting a WM structure. ${ }^{31}$ In neither experiment was evidence of a second conformer found. This matrix effect is particularly remarkable when considered in the light of the authors' $a b$ initio computations which showed that the conformers differed in binding energy by a mere $0.084 \mathrm{kcal} / \mathrm{mol}$. If one assumes that this estimate is closer to the truth, the matrix effect of the two Bakkas et al. reports can be ascribed to a strong, and highly interesting, water-methanol-nitrogen three-body interaction.

Isolated water-methanol dimer complexes have also been identified in a molecular beam by Huisken and Stemmler using an IR dissociation/depletion probe coupled with a time-of-flight mass spectrometer. ${ }^{32}$ The probe used was a line-tunable $\mathrm{CO}_{2}$ laser acting on the $\mathrm{C}-\mathrm{O}$ stretch chromophore, and individual cluster sizes were resolved by scattering off of a perpendicular beam of helium atoms. In previous experiments with methanol dimer, they were able to observe two separate peaks, one to the red and one to the blue of $\mathrm{C}-\mathrm{O}$ fundamental at $1033.9 \mathrm{~cm}^{-1}$, and assigned them to the donor and acceptor subunits, respectively. By analogy, they only observed a red shifted $\mathrm{C}-\mathrm{O}$ stretch for the water-methanol complex at $1027.8 \mathrm{~cm}^{-1}$, in good agreement with the Ar-matrix isolation frequency of $1031.7 \mathrm{~cm}^{-1}$. Thus in the only gas-phase experiment before the present study, evidence was found only for the WM conformer. 


\section{EXPERIMENT}

Studies of the microwave spectrum of $\mathrm{CH}_{3} \mathrm{OH} \cdots \mathrm{H}_{2} \mathrm{O}$ were begun independently and nearly concurrently at Caltech and in the Optical Technology Division at NIST, Gaithersburg. The NIST work stemmed from an on-going effort to measure the rotation-tunneling spectrum of the methanol dimer. ${ }^{33}$ Because these two supersonic jet spectrometers are complementary in several ways, including spectral coverage, a joint effort was mounted to complete this project.

The advantages of each instrument for this work are as follows. The NIST Balle-Flygare-type FTMW spectrometer, described in detail elsewhere, ${ }^{34-36}$ is a high resolution instrument, $\Delta \nu \approx 2 \mathrm{kHz}$, capable of measuring many of the small tunneling splittings associated with more strongly bound vdW complexes, which can be recorded with accurate relative intensities. The instrument is also equipped with a high voltage Stark cell, $\pm 5 \mathrm{kV}$ over $26 \mathrm{~cm}$, that can measure the dipole moments of complexes with high precision. One limitation is that it has a relatively slow scanning rate, $200 \mathrm{MHz}$ per hour or less. The FT cavity can also scan only from 7 $\mathrm{GHz}$ to about $26 \mathrm{GHz}$, and even then the antenna must be manually adjusted to optimize the sensitivity from the lowest to the highest frequency. On the other hand, the Caltech planar supersonic jet/direct absorption microwave spectrometer, also described previously, ${ }^{37,10}$ begins coverage at about $18 \mathrm{GHz}$ and can reach up to $80 \mathrm{GHz}$. This machine has a much lower resolution of a few hundred $\mathrm{kHz}$, but can scan quickly, up to $20 \mathrm{MHz} / \mathrm{min}$ or $1.2 \mathrm{GHz} / \mathrm{h}$, allowing a broad, automated collection of data. The microwave spectrometer is also equipped with a low voltage Stark cell, used here to discriminate between $A$ and $E$ methyl rotor states. ${ }^{38}$

A total of 73 transitions between 21.9 and $55.6 \mathrm{GHz}$ were recorded at Caltech for the $\mathrm{CH}_{3} \mathrm{OH} \cdots \mathrm{H}_{2} \mathrm{O}$ isotopomer which required both methanol and water [Fig. 3(a)]. Initial efforts at scanning were slowed due to the much more numerous methanol dimer lines. The methanol dimer complex has 16 tunneling transitions which, along with transitions from different populated $K_{a}$ manifolds, obscured hundreds of $\mathrm{MHz}$ of spectral coverage, and required a large amount of time to distinguish from the more sparse water-methanol lines. Later on, approximate rotational constants for the methanol dimer from NIST allowed us to selectively avoid these congested areas. ${ }^{33}$ At the same time, 8 transitions corresponding to a methanol + water chemistry were recorded at NIST: 2 between 7.7 and $8.0 \mathrm{GHz}$ and 6 between 15.4 and $16.5 \mathrm{GHz}$. Some tunneling splittings on the order of tens of $\mathrm{kHz}$ were observed in the NIST spectra. Once some of these $\mathrm{CH}_{3} \mathrm{OH} \cdots \mathrm{H}_{2} \mathrm{O}$ transitions were fit to an asymmetric rotor Hamiltonian, transitions from other isotopomers were predicted, and therefore preassigned, from a crude WM structure and observed. These included 3 NIST and 17 Caltech lines for $\mathrm{CH}_{3} \mathrm{OD} \cdots \mathrm{D}_{2} \mathrm{O}, 5$ NIST lines each for $\mathrm{CD}_{3} \mathrm{OH} \cdots \mathrm{H}_{2} \mathrm{O}$ and $\mathrm{CH}_{3} \mathrm{OH} \cdots \mathrm{DOH}$, and 6 NIST lines for ${ }^{13} \mathrm{CH}_{3} \mathrm{OH} \cdots \mathrm{H}_{2} \mathrm{O}$. The Caltech data corresponds to transitions in $J$ from $J=2$ up to $J=6$ or 7 in the $K_{a}=0,1,2$ manifolds; NIST lines were from $J=0, K_{a}=0$ and $J=1, K_{a}=0,1$. The NIST work also measured the following dipole moment
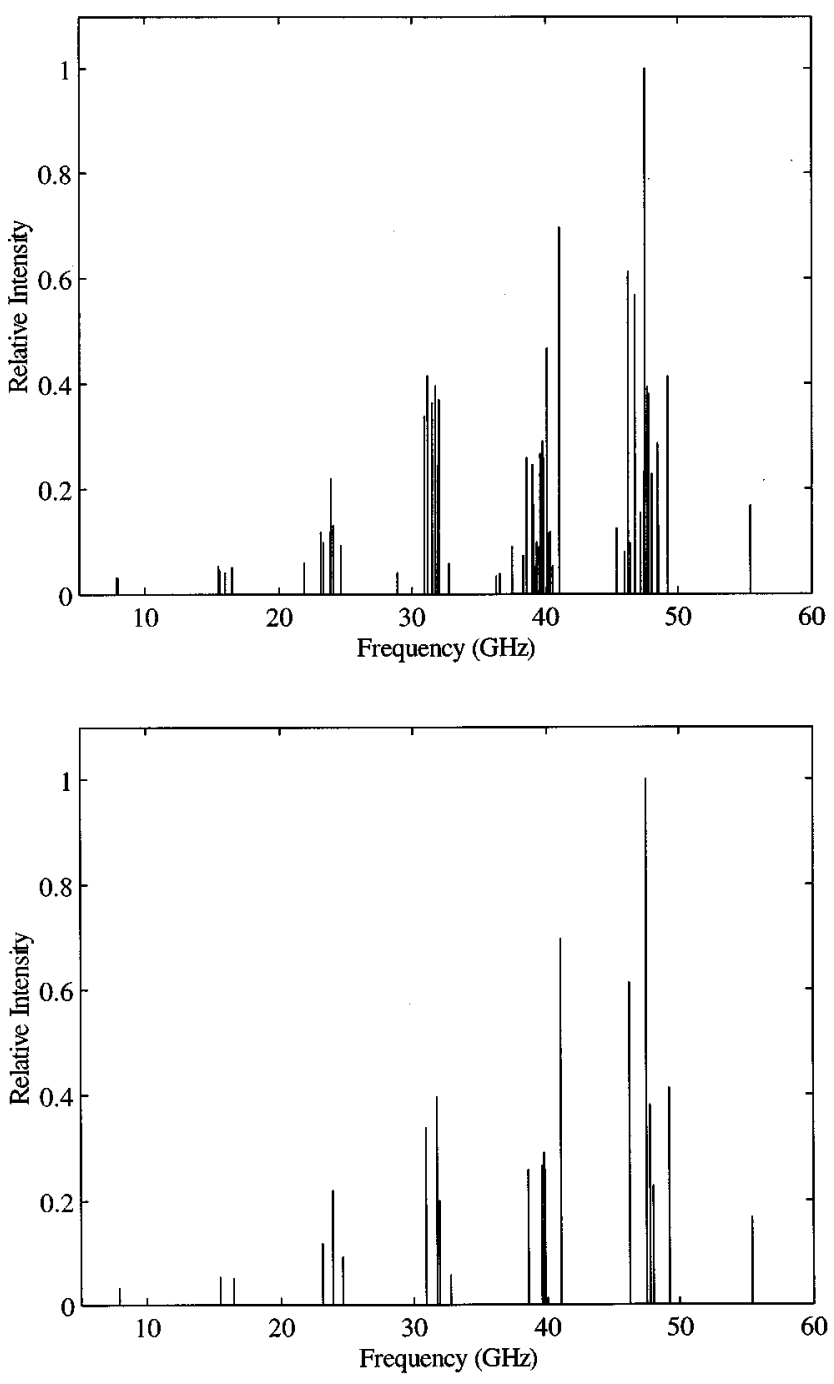

FIG. 3. (a) Observed and (b) assigned $A$ state microwave transitions for the $\mathrm{CH}_{3} \mathrm{OH} \cdots \mathrm{H}_{2} \mathrm{O}$ complex.

components: $\quad \mu_{a}=7.956 \pm 0.03 \times 10^{-30} \mathrm{C} \mathrm{m}$

$\pm 0.008 \mathrm{D}), \quad \mu_{b}=3.636 \pm 0.02 \times 10^{-30} \mathrm{C} \mathrm{m}$

$\pm 0.006 \mathrm{D}), \quad$ and $\quad \mu_{c}=0.43 \pm 0.47 \times 10^{-30} \mathrm{C} \mathrm{m} \quad(0.13$ $\pm 0.14 \mathrm{D}$ ), where the errors listed correspond to one standard deviation uncertainties.

The lines at Caltech were observed with a continuous flow rate of $3.601 / \mathrm{min} \mathrm{Ar} / 6.00 \mathrm{l} / \mathrm{min} \mathrm{Ar}+\mathrm{H}_{2} \mathrm{O} / 0.2701 / \mathrm{min}$ $\mathrm{Ar}+\mathrm{CH}_{3} \mathrm{OH}$. In the NIST experiments, $\mathrm{CH}_{3} \mathrm{OH}$ and $\mathrm{H}_{2} \mathrm{O}$, entrained in separate flows of Ar, were introduced into the cavity through a 1.0 -mm-diam pulsed solenoid valve over 200-400 $\mu$ s durations at rates up to $10 \mathrm{~Hz}$. The total backing pressure in the Caltech experiment was $\sim 2 \mathrm{~atm}$ and for the NIST experiment it was $1 \mathrm{~atm}$. No special attention was given to the purity of the $\mathrm{CH}_{3} \mathrm{OH}$ or $\mathrm{H}_{2} \mathrm{O}$ used; ${ }^{13} \mathrm{C}$ and deuterium enriched samples were obtained from commercial suppliers.

\section{SPECTRAL ANALYSIS}

The initial assignments were greatly facilitated by the much simpler rotational structure in the low quantum num- 
TABLE II. $A$-state rotational transitions $(\mathrm{MHz})$ for $\mathrm{CH}_{3} \mathrm{OH} \cdots \mathrm{H}_{2} \mathrm{O}$ and $\mathrm{CH}_{3} \mathrm{OD} \cdots \mathrm{D}_{2} \mathrm{O}$

\begin{tabular}{|c|c|c|c|c|}
\hline Transition & $\mathrm{CH}_{3} \mathrm{OH} \cdots \mathrm{H}_{2} \mathrm{O}$ & $(o-c)$ & $\mathrm{CH}_{3} \mathrm{OD} \cdots \mathrm{D}_{2} \mathrm{O}$ & $(o-c)$ \\
\hline $1_{01^{-}}-0_{00}$ & 7978.595 & -0.0003 & & \\
\hline $2_{12}-1_{11}$ & 15470.202 & 0.0000 & 14339.873 & -0.0005 \\
\hline $2_{02}-1_{01}$ & 15948.774 & -0.0003 & 14754.546 & 0.0000 \\
\hline $2_{11}-1_{10}$ & 16440.284 & 0.0002 & 15158.690 & -0.0010 \\
\hline $3_{13}-2_{12}$ & 23198.622 & 0.0014 & 21504.37 & 0.18 \\
\hline $3_{03}-2_{02}$ & 23902.179 & 0.0000 & 22114.40 & 0.28 \\
\hline $3_{22}-2_{21}$ & 23924.74 & -1.80 & & \\
\hline $3_{21}-2_{20}$ & 23953.78 & -1.48 & & \\
\hline $3_{12}-2_{11}$ & 24652.00 & -0.02 & 22730.18 & 0.57 \\
\hline $4_{14}-3_{13}$ & 30919.68 & 0.53 & 28662.01 & 0.13 \\
\hline $4_{04}-3_{03}$ & 31831.28 & 0.68 & 29453.22 & 0.55 \\
\hline $4_{23}-3_{22}$ & 31888.30 & -2.48 & & \\
\hline $4_{22}-3_{21}$ & 31960.68 & -1.67 & & \\
\hline $4_{13^{-}}-3_{12}$ & 32855.04 & 1.43 & 30291.31 & 0.94 \\
\hline $5_{15^{-}} 4_{14}$ & 38629.84 & 0.49 & 35811.19 & 0.28 \\
\hline $5_{05^{-}} 4_{04}$ & 39726.96 & 0.83 & 36763.99 & 0.43 \\
\hline $5_{24^{-}} 4_{23}$ & 39842.34 & -3.06 & & \\
\hline $5_{23}-4_{22}$ & 39986.38 & -1.44 & & \\
\hline $5_{14^{-}} 4_{13}$ & 41043.15 & 1.58 & 37838.34 & 0.78 \\
\hline $6_{16^{-}} 5_{15}$ & 46326.26 & -0.62 & 42949.62 & 0.23 \\
\hline $6_{06}-5_{05}$ & 47581.19 & -0.18 & 44040.89 & 0.24 \\
\hline $6_{25}-5_{24}$ & 47784.42 & -3.63 & & \\
\hline $6_{24}-5_{23}$ & 48034.79 & -0.71 & & \\
\hline $6_{15^{-}} 5_{14}$ & 49215.09 & 2.76 & 45366.95 & 0.74 \\
\hline $7_{17}-6_{16}$ & & & $50078.70^{\mathrm{a}}$ & 3.10 \\
\hline $7_{07}-6_{06}$ & 55388.85 & -0.96 & 51277.76 & 0.74 \\
\hline $7_{16^{-}} 6_{15}$ & & & $52870.37^{\mathrm{a}}$ & 6.89 \\
\hline $8_{18^{-}} 7_{17}$ & & & 57187.88 & 0.16 \\
\hline $8_{08}-7_{07}$ & & & 58472.93 & 0.23 \\
\hline
\end{tabular}

${ }^{\mathrm{a}}$ Not fit.

ber NIST data. Only two lines for $\mathrm{CH}_{3} \mathrm{OH} \cdots \mathrm{H}_{2} \mathrm{O}$ were observed in the $J=0 \rightarrow 1$ region, and these were separated by $\sim 200 \mathrm{MHz}$. Stark measurements indicated that the lowerfrequency transition had a first order Stark effect, while the higher-frequency transition had only a second order Stark effect. Internal rotation of three-fold rotors leads to an $A$ symmetry state and a doubly degenerate $E$ symmetry state. The degeneracy of the $E$ state supports a first order Stark effect, and those lines without a first order Stark effect could therefore be assigned to the $A$ symmetry state. Assignments based upon the higher frequency of the two led to a set of lines which could be fit to a standard asymmetric rotor Hamiltonian, including transitions with $J=0$ to 7 and $K_{a}$

TABLE III. $A$-state rotational transitions $(\mathrm{MHz})$ for $\mathrm{CD}_{3} \mathrm{OH} \cdots \mathrm{H}_{2} \mathrm{O}$, $\mathrm{CH}_{3} \mathrm{OH} \cdots \mathrm{DOH}$, and ${ }^{13} \mathrm{CH}_{3} \mathrm{OH} \cdots \mathrm{H}_{2} \mathrm{O}$.

\begin{tabular}{lrrc}
\hline \hline Transition & $\mathrm{CD}_{3} \mathrm{OH} \cdots \mathrm{H}_{2} \mathrm{O}$ & $\mathrm{CH}_{3} \mathrm{OH} \cdots \mathrm{DOH}$ & ${ }^{13} \mathrm{CH}_{3} \mathrm{OH} \cdots \mathrm{H}_{2} \mathrm{O}$ \\
\hline $1_{01} 0_{00}$ & 7384.830 & $(7905.251)$ & 7840.351 \\
$F=0-1$ & & 7905.166 & \\
$F=2-1$ & & 7905.243 & \\
$F=1-1$ & & 7905.291 & \\
$2_{12} 1_{11}$ & 14312.672 & 15334.694 & 15200.679 \\
$2_{02^{-}} 1_{01}$ & 14760.490 & 15802.445 & 15672.362 \\
$2_{11^{-1}} 1_{10}$ & 15214.723 & 16282.568 & 16157.644 \\
$3_{13}-2_{12}$ & & & 22794.393 \\
$3_{03}-2_{02}$ & 22117.889 & 23683.567 & 23487.755 \\
\hline \hline
\end{tabular}

TABLE IV. Fitted spectroscopic parameters for methanol-water isotopomers.

\begin{tabular}{lcc}
\hline \hline & $\mathrm{CH}_{3} \mathrm{OH} \cdots \mathrm{H}_{2} \mathrm{O}$ & $\mathrm{CH}_{3} \mathrm{OD} \cdots \mathrm{D}_{2} \mathrm{O}$ \\
\hline$A(\mathrm{MHz})$ & $28264(54)^{\mathrm{a}}$ & $25695(94)$ \\
$B(\mathrm{MHz})$ & $4232.17(14)$ & $3895.601(16)$ \\
$C(\mathrm{MHz})$ & $3747.01(14)$ & $3485.454(12)$ \\
$\Delta_{J}(\mathrm{kHz})$ & $49.8(8)$ & $57.6(1.0)$ \\
$\Delta_{J K}(\mathrm{kHz})$ & $314(14)$ & $2746(8)$ \\
$\delta_{J}(\mathrm{kHz})$ & $10.7(1.2)$ & $23.0(6)$ \\
\hline \hline
\end{tabular}

${ }^{\mathrm{a}}$ Uncertainties in parentheses are two standard deviations.

$=0,1,2$. These assigned spectra are seen in Fig. 3(b); Table II contains the line list with observed and calculated frequencies, as well as a complementary set of $A$ symmetry state data for the $\mathrm{CH}_{3} \mathrm{OD} \cdots \mathrm{D}_{2} \mathrm{O}$ isotopomer. Table III lists the transitions of the smaller data sets from NIST for $\mathrm{CD}_{3} \mathrm{OH} \cdots \mathrm{H}_{2} \mathrm{O}, \quad \mathrm{CH}_{3} \mathrm{OH} \cdots \mathrm{DOH}$, and ${ }^{13} \mathrm{CH}_{3} \mathrm{OH} \cdots \mathrm{H}_{2} \mathrm{O}$. Tables IV and Tables $\mathrm{V}$ give the corresponding fitted spectroscopic constants for these complexes. Because of the limited data set for the last three complexes, the $A$ rotational constant was not well determined, and fixed at an estimated value in the final fit.

Assignment and fitting of the $E$ state data are not so straightforward, and current progress has given only a tentative assignment. Evidence for the $E$ state is most obvious in the NIST $J=0 \rightarrow 1$ lines, where the lower-frequency component has a first order Stark effect, consistent with an excited internal methyl rotor. A simple $R$-branch progression was found to be built upon this transition, for which $K_{a}=0$. Assignment of the $K_{a} \neq 0 E$ state components is complicated by the fact that the not-too-asymmetric top $A$ symmetry state has nearly degenerate transitions for $K_{a}=2$, which also have pseudo-first order Stark effects at low values of $J$. After complete assignment of the $A$ state data eliminated these as choices, two additional sets of $E$ state lines were identified, each again fitting a separate $R$-branch progression. Stark shifts indicate that these two are characteristic of $K_{a}=1$ states. The tentative assignments are given in Table VI. Initial attempts at fitting more than one progression at a time to a standard internal rotor Hamiltonian have been only partly successful, and will be discussed at greater length below.

TABLE V. Fitted spectroscopic parameters for methanol-water isotopomers, continued. Values of $A$ marked with an asterisk were fixed to structural approximations. Uncertainties in parentheses are two standard deviations.

\begin{tabular}{lccc}
\hline \hline & $\mathrm{CD}_{3} \mathrm{OH} \cdots \mathrm{H}_{2} \mathrm{O}$ & $\mathrm{CH}_{3} \mathrm{OH} \cdots \mathrm{DOH}$ & ${ }^{13} \mathrm{CH}_{3} \mathrm{OH} \cdots \mathrm{H}_{2} \mathrm{O}$ \\
\hline$A(\mathrm{MHz})$ & $23300^{\mathrm{a}}$ & $28000^{\mathrm{a}}$ & $28000^{\mathrm{a}}$ \\
$B(\mathrm{MHz})$ & $3917.017(2)$ & $4189.903(3)$ & $4159.739(2)$ \\
$C(\mathrm{MHz})$ & $3468.056(2)$ & $3715.525(3)$ & $3680.816(2)$ \\
$\Delta_{J}(\mathrm{kHz})$ & $61.1(1)$ & $43.9(1)$ & $50.3(1)$ \\
$\Delta_{J K}(\mathrm{kHz})$ & $498(1)$ & $205(2)$ & $84.8(9)$ \\
$\delta_{J}(\mathrm{kHz})$ & $10.7^{\mathrm{b}}$ & $10.7^{\mathrm{b}}$ & $13.4(5)$ \\
$e Q q_{a a}(\mathrm{D})(\mathrm{kHz})$ & & $167(10)$ & \\
\hline \hline
\end{tabular}

${ }^{\mathrm{a}}$ Not fit.

${ }^{\mathrm{b}}$ Fixed at value for parent isotopic species. 
TABLE VI. Tentative assignments for $\mathrm{CH}_{3} \mathrm{OH} \cdots \mathrm{H}_{2} \mathrm{O} E$ state rotational transitions

\begin{tabular}{|c|c|}
\hline Assignment & Frequency $(\mathrm{MHz})$ \\
\hline $1_{01^{-}}-0_{00}$ & 7763.750 \\
\hline $2_{02}-1_{01}$ & 15541.721 \\
\hline $3_{03}-2_{02}$ & 23342.88 \\
\hline $4_{04}-3_{03}$ & 31164.40 \\
\hline $5_{05^{-4}} 4_{04}$ & 39000.56 \\
\hline $6_{06}-5_{05}$ & 46838.52 \\
\hline $2_{12}-1_{11}$ & 15956.630 \\
\hline $3_{13}-2_{12}$ & 23812.08 \\
\hline $4_{14^{-}}-3_{13}$ & 31545.84 \\
\hline $5_{15^{-}} 4_{14}$ & 39173.76 \\
\hline $6_{16^{-}} 5_{15}$ & 46732.50 \\
\hline $2_{11}-1_{10}$ & 15958.460 \\
\hline $3_{12}-2_{11}$ & 24000.92 \\
\hline $4_{13}-3_{12}$ & 32103.44 \\
\hline $5_{14}-4_{13}$ & 40247.31 \\
\hline $6_{15}-5_{14}$ & 48394.68 \\
\hline
\end{tabular}

\section{STRUCTURAL ANALYSIS}

As emphasized above, structure, especially the gross features which determine proton donor and acceptor roles, is of key importance in this initial microwave study of the watermethanol complex. The simple Euler-type angular coordinates widely employed in the analyses of other, smaller, dimers, were not used in this structural analysis. As the subunits grow larger, these do not correspond well with logical intermolecular normal modes of the complex. Instead, the structure is parameterized in the internal coordinates developed by Thompson, where each atom is specified by its orientation with respect to the last three specified atoms. ${ }^{39}$ The coordinates consist of a bond length, a bond angle, and a torsional angle. Of course, the first three atoms have a reduced number of coordinates. The structure fitting routine used was STRFTQ, written by Schwendeman ${ }^{40}$ and modified by Lovas, which allows multiple isotopomers to be fit simultaneously. There are several advantages to using the internal coordinate system in combination with this fitting routine. First, the number of parameters to be fit is reduced from similar programs using principal axis coordinates. Also, symmetry can be specified within a molecule. For example, if fitting the HCF angle in methyl fluoride, one could specify that all three hydrogens form the same $\mathrm{HCF}$ angle and that they be adjusted symmetrically during the fit. Finally, ghost atoms with zero mass can be used to simplify the inputed structure. For example, in methanol, the methyl group tilts $3.3^{\circ}$ away from the hydroxyl group, meaning that the $C_{3}$ symmetry axis is not parallel with the $\mathrm{C}-\mathrm{O}$ bond. The movement of all three methyl hydrogens can be accommodated in one parameter by first adding a ghost atom, bonded to the carbon atom, at the center of the triangle formed by the hydrogens; the hydrogens are then specified relative to the ghost atom.

The starting point for the fit, as well as the algorithm for the fit itself, contains some assumptions which are inherently subjective. Below are the assumptions used in this structural analysis and their justifications.

(1) Assume ground state geometries for the monomer structures (Harmony et $\mathrm{al}^{41}$ ).

(2) Angle $\mathrm{COH}$ of methanol $=108.5^{\circ} \approx 109.5^{\circ}$, so assume a starting geometry of $\mathrm{C}, \mathrm{H}$, and the two lone pairs about oxygen that is tetrahedral.

(3) Assume the intermolecular starting geometry of both MW and WM are that of water-water dimer. For this geometry take structure I from Odutola and Dyke, since this has the lowest standard deviation and its low value of $\chi_{a}$ is consistent with Assumption $2^{14}$ (see Fig. 1).

(4) Since we do not know a priori how the methyl group in $\mathrm{CH}_{3} \mathrm{OH}$ affects the geometry of the lone pairs, use Assumption 2 to determine $\theta_{a}$ for $\mathrm{WM}$, and use angle $\mathrm{O}_{1} \mathrm{O}_{2} \mathrm{H}_{5}$ from WM to fix angle $\mathrm{O}_{2} \mathrm{O}_{1} \mathrm{H}_{1}$ in MW (i.e., $0.74^{\circ}$ ).

A methanol geometry was first computed in internal coordinates, using the ghost atom as described above. Then, the water subunits of the two different conformers were specified relative to the methanol geometry. The final so-called $Z$-matrix for each conformer is given in Table VII.

A number of structural fits were run during the course of this work in order to obtain predictions for different isotopomers, and also to check preliminary findings for the donor/ acceptor roles. In the fits, $B$ and $C$ rotational constants were used along with the well-determined $A$ rotational constants for $\mathrm{CH}_{3} \mathrm{OH} \cdots \mathrm{H}_{2} \mathrm{O}$ and $\mathrm{CH}_{3} \mathrm{OD} \cdots \mathrm{D}_{2} \mathrm{O}$. Three parameters were fit for the two different conformers: the hydrogen bond distance $R_{\mathrm{O} \cdots \mathrm{H}}$; the lone pair geometry of the acceptor oxygen, $\theta_{\mathrm{O}}$, to correct for the tetrahedral assumption; and the torsional angle of the water, $\phi_{\mathrm{H}_{2} \mathrm{O}}$, about the hydrogen bond. These correspond to $R_{\mathrm{OC}}$ (less the $\mathrm{O}-\mathrm{H}$ bond length), $\theta_{a}$, and $\chi_{a}$ in the water dimer structure.

All pairings of isotopomeric fits unanimously favored the WM conformer as measured by the residual error of the principal moments of inertia, giving definitive confirmation to the results of the argon matrix and IR photodepletion experiments. The most conclusive example of the structural preference comes from the data for the $\mathrm{CH}_{3} \mathrm{OH} \cdots \mathrm{DOH}$ isotopomer. Due to the similar positions of most of the atoms about the centers of mass for the two conformers, isotopic substitution produced small, but consistent, preferences for WM. But the $\mathrm{CH}_{3} \mathrm{OH} \cdots \mathrm{DOH}$ isotopomer substitutes singly the one atomic position most sensitive to the change in conformation-this D atom is either on the outside of the complex, $>2 \AA$ from the center-of-mass in MW, or it is on the inside (due to greater D-binding zero point energy) of complex, $<1 \AA$ away from the center-of-mass for WM. This is best shown in the following set of fits. The other four isotopomers studied are fit to each conformer separately, with the residual of 0.243 u $\AA^{2}$ (units of moments of inertia) for WM, more than six times smaller than the $1.52 \mathrm{u} \AA^{2}$ for MW. Even more conclusive is the prediction of rotational constants for the sensitive $\mathrm{CH}_{3} \mathrm{OH} \cdots \mathrm{DOH}$ isotopomer. The error in $B+C$ is only $5.42 \mathrm{MHz}$ for the WM fit structure, while $B+C$ differs by $246.37 \mathrm{MHz}$ for the MW predictions. 
TABLE VII. Z-matrix coordinates for MW and WM.

\begin{tabular}{|c|c|c|c|c|c|c|}
\hline Atom & Atom\# & $\begin{array}{l}\text { Connected } \\
\text { to atom\# }\end{array}$ & Unit mass & $r$ & $\alpha$ & $\beta$ \\
\hline \multicolumn{7}{|c|}{$Z$-matrix coordinates for $\mathrm{CH}_{3} \mathrm{OH}$} \\
\hline $\mathrm{H}_{1}$ & 1 & & 1. & & & \\
\hline $\mathrm{O}_{1}$ & 2 & 1 & 16. & 0.945 & & \\
\hline $\mathrm{C}$ & 3 & 2 & 12. & 1.425 & 108.5 & \\
\hline G & 4 & 3 & 0. & 0.380 & 176.7 & 180 \\
\hline $\mathrm{H}_{2}$ & 5 & 4 & 1. & 1.026 & 90.0 & 0 \\
\hline $\mathrm{H}_{3}$ & 6 & 4 & 1. & 1.023 & 90.0 & 120 \\
\hline $\mathrm{H}_{4}$ & 7 & 4 & 1. & 1.023 & 90.0 & -120 \\
\hline \multicolumn{7}{|c|}{$Z$-matrix coordinates for MW } \\
\hline $\mathrm{O}_{2}$ & 8 & 1 & 16. & 2.032 & 179.0 & 180 \\
\hline $\mathrm{H}_{5}$ & 9 & 8 & 1. & 0.972 & 122.0 & -68.67 \\
\hline $\mathrm{H}_{6}$ & 10 & 8 & 1. & 0.972 & 122.0 & 68.67 \\
\hline \multicolumn{7}{|c|}{$Z$-matrix coordinates for WM } \\
\hline $\mathrm{H}_{5}$ & 8 & 2 & 1. & 2.005 & 109.0 & 120 \\
\hline $\mathrm{O}_{2}$ & 9 & 8 & 16. & 0.972 & 179.0 & -60 \\
\hline $\mathrm{H}_{6}$ & 10 & 9 & 1. & 0.972 & 104.5 & 180 \\
\hline
\end{tabular}

The resulting structure is very similar to that of water dimer, as expected. The $R_{\mathrm{OO}}$ bond length of 2.997 $\pm 0.009 \AA$ is, surprisingly, longer, but only by $0.02 \AA$, than that for the water dimer, and the bond angle is nearly linear at $179 \pm 1^{\circ}$. The torsional angle was not well-fit, and on the average gave a structure close to bifurcation of the methanol $\mathrm{COH}$ angle, again much like water dimer.

\section{DISCUSSION AND CONCLUSIONS}

Ruoff $e t$ al. have shown that relaxation of weakly bound clusters with multiple conformations to the lowest ground state energy conformer is efficient and near complete in seeded supersonic jet expansions of argon. ${ }^{42}$ In light of this, the results of the structural analysis for the present data set are unambiguous - the lower-energy conformer of the water-methanol dimer has a water-donor, methanolacceptor structure, and has a bonding geometry similar to that of the water dimer. Furthermore, the absence of any lines assignable to a MW structure from the $T_{\text {rot }} \approx 5 \mathrm{~K}$ supersonic expansion suggests that this second conformer is $\geqslant 10-15 \mathrm{~cm}^{-1}$ higher in ground state energy relative to the more stable WM conformer. The magnitude of the error for the measurement of $\mu_{c}=0.43 \pm 0.47 \times 10^{-30} \mathrm{C} \mathrm{m} \quad(0.13$ $\pm 0.14 \mathrm{D})$, along with the substantial values of $\mu_{a}$ and $\mu_{b}$, is consistent with the dipole moments expected for either conformer. Beyond the structural analysis, these preliminary spectra raise questions about the effects of intermolecular wide-amplitude motions, especially the internal rotations of the methyl group.

The estimates of Hougen and Ohashi ${ }^{25}$ predict four significant tunneling splittings for the MW conformer and eight for WM. The $A-E$ splitting is very large, hundreds of $\mathrm{MHz}$, and observable for all values of $J$ and $K$ measured. The NIST spectra also recorded a much smaller splitting, 30-100 $\mathrm{kHz}$, for all $A$ state lines of all isotopomers. This gives a total of only four tunneling components, in seeming contradiction to the structural analysis results. However, these splittings were predicted to have "top-to-top, bottom-to-bottom" $a$-type selection rules, and so the effects of the splittings might not be apparent in the present data, as was the case for two of the tunneling components in the methanol dimer. ${ }^{33}$

A search up to $60 \mathrm{GHz}$, more than twice the structural value of $A$, turned up no identifiable $b$-type transitions. These spectra, which should have "top-to-bottom, bottomto-top" selection rules, have a large dipole moment component, and should have many strong $Q$-branches. However, as previously seen for $\mathrm{H}_{3} \mathrm{~N} \cdots \mathrm{H}_{2} \mathrm{O},{ }^{10}$ internal rotation can increase the frequency of the $b$-type $\left(\Delta K_{a, c}= \pm 1\right)$ spectra to more than four times that predicted by structure alone. Once these $b$-type rotation-tunneling spectra are measured, the tunneling splittings can be calculated from the difference in rotational constants for each symmetry state.

Several hydrogen-bonded complexes containing methyl rotors have been previously examined in the literature: $\mathrm{CH}_{3} \mathrm{OH} \cdots \mathrm{NH}_{2} \mathrm{CHO},{ }^{2} \quad \mathrm{CH}_{3} \mathrm{OH} \cdots \mathrm{CO},{ }^{43} \quad \mathrm{CH}_{3} \mathrm{OH} \cdots \mathrm{SO}_{2},{ }^{44}$ $\left(\mathrm{CH}_{3} \mathrm{OH}\right)_{2},{ }^{33} \mathrm{CH}_{3} \mathrm{OH} \cdots \mathrm{Ar},{ }^{35,45}$ and $\mathrm{CH}_{3} \mathrm{OH} \cdots \mathrm{HCl}{ }^{46,47}$ For each of these species, an internal rotor Hamiltonian has been used successfully in combined fits of $A$ and $E$ state data [and in the case of $\left(\mathrm{CH}_{3} \mathrm{OH}\right)_{2}, A, E$, and $G$ states]. Interestingly, in each case the best fit of the $V_{3}$ internal rotation terms yields an apparent lowering of the barrier for the rotation of the methyl top against the hydroxyl "frame." From preliminary internal rotor fits using the assignments presented in Table VI, we also find a similar behavior in water-methanol, with the best fits resulting in $V_{3}$ coefficients of $\sim 60-65 \mathrm{~cm}^{-1}$, in contrast to the value of $\sim 375 \mathrm{~cm}^{-1}$ for free methanol.

Recently, Fraser et al., ${ }^{48}$ followed by Kuczkowski and co-workers, ${ }^{44,45,47}$ studied in detail the barrier to internal methyl rotation for weakly bound complexes containing methanol. They find that the degree of splitting between the $A$ and $E$ torsional energy levels, and hence the perturbation to the rotational Hamiltonian, is not merely dependent on the bar- 
rier to methyl rotation, but rather results primarily from the coupling of the methyl rotation with the internal rotation of the methanol subunit about one of its molecular axes. In the $\mathrm{CH}_{3} \mathrm{OH} \cdots$ Ar dimer, for example, free rotation of the $\mathrm{CH}_{3} \mathrm{OH}$ subunit, similar to that of $\mathrm{H}_{2} \mathrm{O}$ in $\mathrm{Ar} \cdots \mathrm{H}_{2} \mathrm{O},{ }^{49}$ is suspected to couple to the overall rotation of the complex as well as to the internal rotation.

In the complexes previously considered for which the hydroxyl group of methanol was the hydrogen-bond donor, the $A-E$ splitting correlated with the rigidity of this bond. This in turn hinders the internal rotation of the methanol subunit. Mathematical modeling of this behavior was carried out by adding a $v_{1}(1-\cos \theta) / 2$ term to the large amplitude Hamiltonian, where $\theta$ is the rotation angle of the $\mathrm{OH}$ defined with respect to the plane formed by $a$ axis of methanol and the center-of-mass of the binding partner in the complex, and $v_{1}$ is the first coefficient of a one-dimensional Fourier expansion of the true potential. This approximate treatment of the large amplitude motion naturally explains the apparent lowering of the methyl group $V_{3}$ barrier, and can be used to estimate the magnitude of $v_{1}$.

For example, in the $\mathrm{CH}_{3} \mathrm{OH} \cdots \mathrm{HCl}$ complex, initial studies by Cope et al. found a series of $A$ state transitions which were well fit to an asymmetric rotor Hamiltonian; a second $R$-branch progression was also observed to the red of the $K_{a}=0$ lines, much as for the water-methanol data presented here. Another similarity between these results and the present is the large change in $\bar{B}=(B+C): 67 \mathrm{MHz}$, or $1.43 \%$, for $\mathrm{CH}_{3} \mathrm{O}(\mathrm{H}) \cdots \mathrm{HCl}$ and $215 \mathrm{MHz}$, or $2.69 \%$, for $\mathrm{CH}_{3} \mathrm{OH} \cdots \mathrm{H}_{2} \mathrm{O}$ as traced by the $J=1 \rightarrow 2, K_{a}=0$ transition. Cope et al. ascribed this second set of lines to a low-lying vibrational state such as that involved with the lone pair interchange motion, but a recent study by Tan et ll $^{47}$ has definitively assigned these transitions to the $E$ internal rotor state. Detailed fits using a modified Fraser et al. ${ }^{48}$ librational Hamiltonian produced a $V_{3}$ barrier of 74(1) $\mathrm{cm}^{-1}$ and a $v_{1}$ term of $155(5) \mathrm{cm}^{-1}$. Similar complete assignments of the $E$ state water-methanol transitions and cofitting with the $A$ state transitions to the appropriate internal rotor Hamiltonian should yield much information about the IPS. In particular, for cases such as water-methanol, where $\mathrm{CH}_{3} \mathrm{OH}$ can act either as a hydrogen-bond acceptor or donor, this approach can, in principle, be used to provide an estimate of the energetic differences between the two topologies of the complex. $^{47}$

It should be noted, however, that the true potential may well be poorly approximated by a one term $\cos (\theta)$ expansion in cases where the methanol serves as the proton acceptor. Indeed, for water-methanol the excited internal rotor motion coupled to the lone pair interchange is very similar to the combined $n=1 \rightarrow 4$ and $n=1 \rightarrow 10$ tunneling motions described by Hougen and Ohashi. ${ }^{25}$ This leads to a large amplitude librational potential for the methanol which is very flat for orientations which direct one or both of the lone pairs toward the hydrogen bond donor, and to an overall potential that is likely to contain a great deal of $\cos (3 \theta)$ character.

More quantitative constraints on the nature of the watermethanol IPS await the measurement of its gas-phase far- infrared spectrum, and the tremendous previous body of experimental and theoretical work on the water dimer again provides a useful starting point for a consideration of the intermolecular dynamics of water-methanol. Due to the large difference in the librational moments of inertia, separation of the six intermolecular degrees of freedom into distinct donor/acceptor vibrational modes should be a much better approximation for water-methanol than for the water dimer, for which recent high resolution far-infrared spectra ${ }^{50}$ have revealed breakdowns in the high barrier generalized internal axis method so successfully used for the ground state of $\left(\mathrm{H}_{2} \mathrm{O}\right)_{2} \cdot 1,21,22$

In this limit, the out-of-plane and in-plane bends of the donor water should be the stiffest, highest-frequency modes. Various harmonic theoretical estimates place these modes near 550-600 and 350-380 $\mathrm{cm}^{-1}$, respectively, as compared to matrix isolation frequencies of 520 and $320 \mathrm{~cm}^{-1}$. Similar locations can be expected for water-methanol. Studies of these bands are, in principle, possible with lead salt diode lasers, but the poor output power and tuning characteristics of these devices in the $300-500 \mathrm{~cm}^{-1}$ region make such experiments a difficult task at present.

The hydrogen-bond stretching mode is likely to lie between the water librational modes and those involving methanol. A pseudo-diatomic calculation of the stretching mode location using the observed rotational and centrifugal distortion constants results in harmonic frequencies near $75 \mathrm{~cm}^{-1}$ for the parent isotopomer. Similar estimates for the water dimer result in stretching frequencies near $145 \mathrm{~cm}^{-1}$. The lowest-frequency modes should involve either torsional or vibrational modes that are tied primarily to methanol subunit along with the torsion of the donor and acceptor subunits with respect to each other. Simple scaling by the relative moments of inertia between the water dimer and watermethanol results in harmonic frequencies below $40-50 \mathrm{~cm}^{-1}$ for these vibrations. Each of these modes should be observable with current tunable far-infrared laser sideband spectrometers, and should provide new insights into the tunneling dynamics and relative donor-acceptor energetics of watermethanol. Efforts to locate these modes and to complete the assignment of the microwave spectra presented here are currently underway.

\section{ACKNOWLEDGMENTS}

This research was supported in part by a grant from the the National Science Foundation (CHEM-9415488). We thank J. T. Hougen and N. Ohashi for discussions about their work on the permutation-inversion theory of the watermethanol dimer.

${ }^{1}$ L. H. Coudert, F. J. Lovas, R. D. Suenram, and J. T. Hougen, J. Chem. Phys. 87, 6290 (1987).

${ }^{2}$ F. J. Lovas, R. D. Suenram, G. T. Fraser, C. W. Gillies, and J. Zozom, J. Chem. Phys. 88, 722 (1988).

${ }^{3}$ G. T. Fraser, R. D. Suenram, and L. H. Coudert, J. Chem. Phys. 90, 6077 (1989).

${ }^{4}$ R. D. Suenram, G. T. Fraser, and F. J. Lovas, J. Mol. Spectrosc. 138, 440 (1989). 
${ }^{5}$ H. O. Leung, M. D. Marshall, R. D. Suenram, and F. J. Lovas, J. Chem. Phys. 90, 700 (1989).

${ }^{6}$ D. Yaron, K. I. Peterson, D. Zolandz, W. Klemperer, F. J. Lovas, and R. D. Suenram, J. Chem. Phys. 7095, 92 (1990).

${ }^{7}$ G. T. Fraser, F. J. Lovas, R. D. Suenram, and K. Matsumura, J. Mol. Spectrosc. 144, 97 (1990).

${ }^{8}$ S. Suzuki, R. E. Bumgarner, P. A. Stockman, P. G. Green, and G. A. Blake, J. Chem. Phys. 94, 824 (1991).

${ }^{9}$ R. E. Bumgarner, S. Suzuki, P. A. Stockman, P. G. Green, and G. A. Blake, Chem. Phys. Lett. 176, 123 (1991).

${ }^{10}$ P. A. Stockman, R. E. Bumgarner, S. Suzuki, and G. A. Blake, J. Chem. Phys. 96, 2496 (1992).

${ }^{11}$ D. W. Steyert, M. J. Elrod, and R. J. Saykally, J. Chem. Phys. 99, 7431 (1993).

${ }^{12}$ M. Matsumoto, Y. Takaoka, and Y. Kataoka, J. Chem. Phys. 98, 1464 (1993)

${ }^{13}$ H. Tanaka, J. Walsh, and K. E. Gubbins, Mol. Phys. 76, 1221 (1992).

${ }^{14}$ J. A. Odutola and T. R. Dyke, J. Chem. Phys. 72, 5062 (1980).

${ }^{15}$ S. Kuwajima and A. Warshel, J. Phys. Chem. 94, 460 (1990).

${ }^{16}$ W. Rijks and P. E. S. Wormer, J. Chem. Phys. 90, 6507 (1989); E. C. Vauthier, V. Barone, and S. Fliszár, Can. J. Chem. 68, 1233 (1990).

${ }^{17}$ A. McIlroy, R. Lascola, C. M. Lovejoy, and D. J. Nesbitt, J. Phys. Chem. 95, 2636 (1991).

${ }^{18}$ N. Pugliano and R. J. Saykally, J. Chem. Phys. 96, 1832 (1992).

${ }^{19}$ N. Pugliano, J. D. Cruzan, J. G. Loeser, and R. J. Saykally, J. Chem. Phys. 98, 6600 (1993).

${ }^{20}$ J. T. Hougen, J. Mol. Spectrosc. 114, 395 (1985).

${ }^{21}$ L. H. Coudert and J. T. Hougen, J. Mol. Spectrosc. 130, 86 (1988).

${ }^{22}$ L. H. Coudert and J. T. Hougen, J. Mol. Spectrosc. 139, 259 (1990)

${ }^{23}$ M. J. Elrod, R. J. Saykally, A. R. Cooper, and J. M. Hutson, Mol. Phys. 81, 579 (1994).

${ }^{24}$ L. Dore, R. C. Cohen, C. A. Schmuttenmaer, K. L. Busarow, M. J. Elrod, J. G. Loeser, and R. J. Saykally, J. Chem. Phys. 100, 863 (1994).

${ }^{25}$ J. T. Hougen and N. Ohashi, J. Mol. Spectrosc. 159, 363 (1993).

${ }^{26}$ J. E. Del Bene, J. Chem. Phys. 55, 4633 (1971).

${ }^{27}$ Y.-C. Tse, M. D. Newton, and L. C. Allen, Chem. Phys. Lett. 75, 350 (1980).

${ }^{28}$ G. Bolis, E. Clementi, D. H. Wertz, H. A. Scheraga, and C. Tosi, J. Phys. Chem. 105, 355 (1983).
${ }^{29}$ S. Kim, M. S. Jhon, and H. A. Scheraga, J. Phys. Chem. 92, 7216 (1988).

${ }^{30}$ N. Bakkas, Y. Bouteiler, A. Bouteiller, J. P. Perchard, and S. Racine, J. Chem. Phys. 99, 3335 (1993).

${ }^{31}$ N. Bakkas, Y. Bouteiler, A. Bouteiller, J. P. Perchard, and S. Racine, Chem. Phys. Lett. 232, 90 (1995).

${ }^{32}$ F. Huisken and M. Stemmler, Chem. Phys. Lett. 180, 332 (1991).

${ }^{33}$ F. J. Lovas, S. P. Belov, M. Y. Tretyakov, W. Stahl, and R. D. Suenram, J. Mol. Spectrosc. 170, 478 (1995).

${ }^{34}$ F. J. Lovas and R. D. Suenram, J. Chem. Phys. 87, 2010 (1987).

${ }^{35}$ R. D. Suenram, F. J. Lovas, G. T. Gillies, J. Z. Gillies, and M. Onda, J. Mol. Spectrosc. 137, 127 (1989).

${ }^{36}$ F. J. Lovas, N. Zobov, G. T. Fraser, and R. D. Suenram, J. Mol. Spectrosc. 171, 189 (1995).

${ }^{37}$ R. E. Bumgarner and G. A. Blake, Chem. Phys. Lett. 161, 308 (1989).

${ }^{38}$ S. Suzuki, Ph.D. thesis, California Institute of Technology, 1996.

${ }^{39}$ H. B. Thompson, J. Chem. Phys. 47, 3407 (1967).

${ }^{40}$ R. H. Schwendeman, Critical Evaluation of Chemical and Physical Structural Information (National Academy of Sciences, Washington, D.C., 1972).

${ }^{41}$ M. D. Harmony, V. W. Laurie, R. L. Kuczkowski, R. H. Schwendeman, D. A. Ramsay, F. J. Lovas, W. J. Lafferty, and A. G. Maki, J. Phys. Chem. Ref. Data 8, 619 (1979).

${ }^{42}$ R. S. Ruoff, T. D. Klots, T. Emilsson, and H. S. Gutowsky, J. Chem. Phys. 93, 3142 (1990).

${ }^{43}$ F. J. Lovas, S. P. Belov, J. Ortigoso, and R. D. Suenram, J. Mol. Spectrosc. 167, 191 (1994).

${ }^{44}$ L. Sun, X. Q. Tan, J. J. Oh, and R. L. Kuczkowski, J. Chem. Phys. 103, 6440 (1995)

${ }^{45}$ X. Q. Tan, L. Sun, and R. J. Kuczkowski, J. Mol. Spectrosc. 171, 256 (1995).

${ }^{46}$ P. Cope, A. C. Legon, and D. J. Millen, Chem. Phys. Lett. 112, 59 (1984).

${ }^{47}$ X. Q. Tan, I. I. Ioannou, and R. J. Kuczkowski, J. Mol. Struct. 356, 105 (1995).

${ }^{48}$ G. T. Fraser, F. J. Lovas, and R. D. Suenram, J. Mol. Spectrosc. 167, 231 (1994).

${ }^{49}$ R. Carl Cohen, Ph.D. thesis, University of California, Berkeley, 1992.

${ }^{50}$ K. Liu, J. D. Cruzan, and R. J. Saykally, Science 271, 929 (1996). 